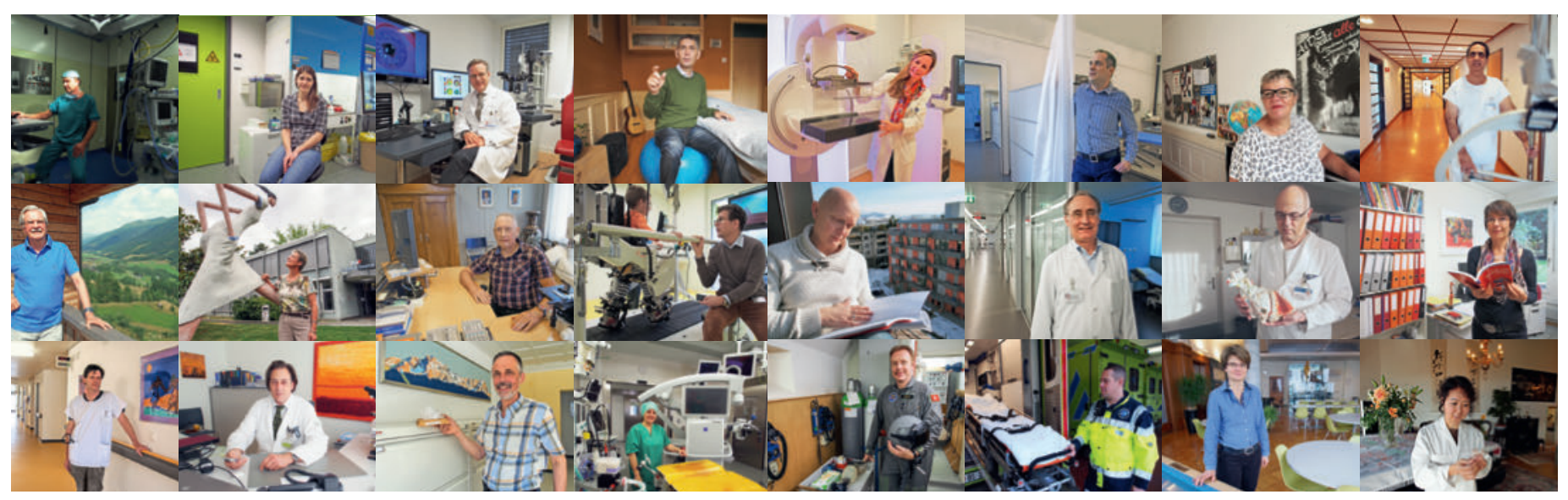

\title{
Gesichter des Gesundheitswesens
}

\section{Daniel Lüthi}

Freier Journalist und Fotograf, Medientrainer, Bern

Seit März 2010 und bis Ende Jahr publiziert die Schweizerische Ärztezeitung jeden Monat die Rubrik "Begegnung mit ...». Es sind Begegnungen von Daniel Lüthi mit Menschen, die im Gesundheitswesen tätig sind. Der Autor gibt diesen Menschen mit seinen Porträts ein Gesicht. Nach seinem 1. Buch Begegnungen mit dem Gesundheitswesen (2013) erscheint jetzt Band 2, wiederum mit 32 Porträts von Ärztinnen und Ärzten, aber auch von anderen "Gesundheitswesen». In diesem Artikel fasst der Autor seine Erfahrungen mit der Serie und seine Gedanken zum Buch zusammen.

Es waren immer lange Gespräche, und eigentlich ging es immer um Gesundheit und Krankheit, Leben und Tod, Geburt und Sterben. Wer sich aufmacht, das Gesundheitswesen kennenzulernen, kommt Existentiellem auf die Spur. Vor allem aber lernt er Menschen kennen, die sich mit grossem Engagement und viel Herzblut für das Wohl anderer einsetzen.

Jedes dieser Gespräche war ein gemeinsamer Weg. Ihn gehen zu dürfen, ist ein Privileg. Die Schweizerische
Ärztezeitung SÄZ hat diesen Weg geebnet, hat den Rahmen für diese Begegnungen geschaffen. Dafür gebührt ihr Anerkennung und Dank. Bruno Kesseli, Chefredaktor und Arzt, war immer davon überzeugt, dass sich das Gesundheitswesen über Zahlen und Statistiken allein nicht definieren lässt. Dass es dieses ominöse Wesen verdient hat, nicht bloss als ökonomischer Faktor wahrgenommen zu werden. Seit Jahren und mit Erfolg machen er und sein Team das "gelbe Heftli» zu

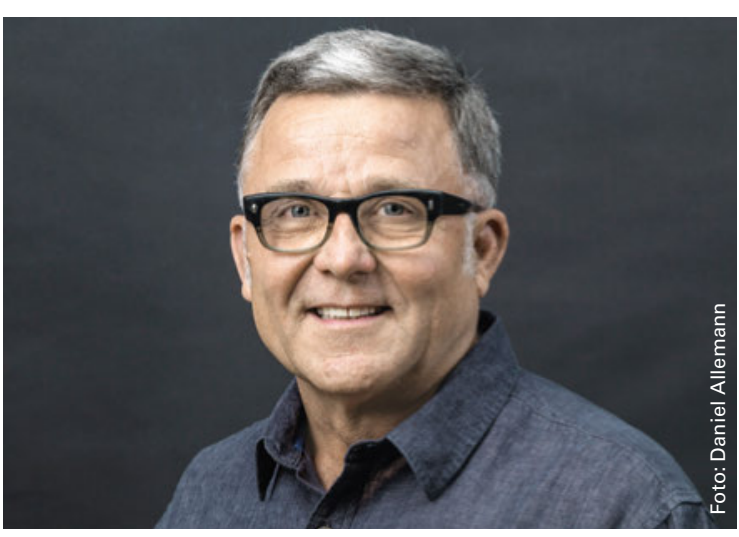

\section{Der Autor}

Daniel Lüthi, geboren 1958 in Bern. Er arbeitet als selbständiger Journalist, Medientrainer, Moderator, Dozent und Autor. Er ist Inhaber und Geschäftsführer der Firma «DL Kommunikation» und lebt in Bern (www.dlkommunikation.ch). 


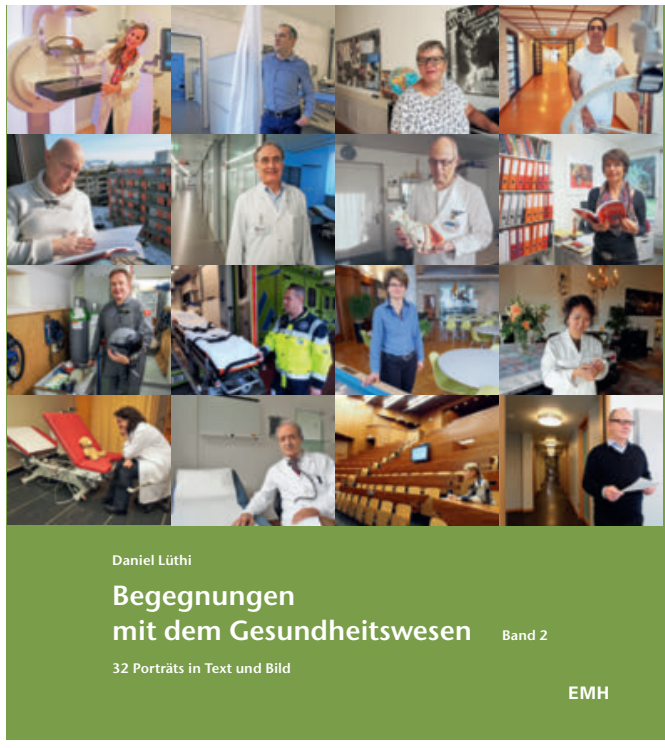

Daniel Lüthi

Begegnungen mit dem Gesundheitswesen Band 2

Muttenz: EMH Schweiz. Ärzteverlag AG; 2016.

Broschiert. $28 \mathrm{CHF}$.

ISBN 978-3-03754-094-7

einer Publikation, die mehr bietet als Grafiken und Diagramme, Todesanzeigen und Nachrichten zu Praxiseröffnungen.

Jetzt sind es rigorose Sparvorgaben, die ihn dazu zwingen, im redaktionellen Teil Bewährtes und Wünschbares zu streichen, und deshalb auch die Rubrik «Begegnung mit ...» als Serie auslaufen zu lassen. So gesehen ist dieses Buch auch ein Schlusspunkt. Vor allem aber ist es ein Höhepunkt.

Höhepunkt deshalb, weil hier zwischen zwei Buchdeckeln 32 Kurzgeschichten, die in diesem Heft in den vergangenen drei Jahren einzeln publiziert waren, in einer Art Gesamtwerk vereint sind. Damit werden sie nachhaltiger. Für einen Journalisten ist dies ein ganz besonderes Erlebnis: Texte und Bilder, die flüchtig waren und längst im Altpapier gelandet sind, erhalten einen neuen Wert. Und damit die Geschichten von Menschen, die in ihrem Fachgebiet (und oft weit darüber hinaus) Ausserordentliches leisten. Die bereit sind, davon zu erzählen. Und dabei auch laut über sich selber nachdenken. Dass ein Medium Platz für solche
Geschichten schafft, schätzen die Porträtierten ebenso wie andere, die sie via diese Porträts kennenlernen. Bemerkenswert ist sicher, dass der Blick über den Gartenzaun immer möglich war. So kommen in diesem Buch denn nicht bloss Chefärztinnen und Chefärzte zu Wort, sondern beispielsweise auch ein Apotheker, der Cannabis verschreiben darf, oder ein Pakistaner, der in einem Universitätsspital Patientinnen und Patienten transportiert. Er sagt: «Ich will mit Herz arbeiten.» Immer geht es in diesen Porträts um Gesundheit und Krankheit, Leben und Tod, wie gesagt. Aber auch um mehr Druck und weniger Zeit. Umso schöner, wenn sich vielbeschäftigte Leute zwei Stunden für ein Gespräch reservieren. Alle sagen zwar, dass dies in ihrem beruflichen Umfeld - besonders im Umgang mit Patientinnen und Patienten - das Wichtigste wäre. Aber eben.

Und viele von denen, die jammern, pflegen zu sagen: Wir jammern auf hohem Niveau. Einige Ärztinnen und Ärzte, die im Buch vorkommen, waren oder sind auch in Entwicklungsländern tätig. Selber habe ich lange in Bolivien gelebt. Solche Erfahrungen verstärken das Bewusstsein - und die Dankbarkeit - dafür, wie privilegiert wir hierzulande gerade bei den Rahmenbedingungen sind, die die Medizin bietet. Hohe technische Standards stehen im Vordergrund. Anderes rückt damit allzu oft in den Hintergrund. Oder wie es eine Hausärztin im ersten Band sagt: «In der Sprechstunde ist man vom Sprechen weggekommen.»

Sprechen ist das eine, Zuhören das andere. Wer Geschichten erzählt, verbindet Menschen.

Und wer liest, hört zu.

Geniessen Sie im Zeitalter der Elektronik das Erlebnis, ein Buch zu lesen und Gesichter des Gesundheitswesens kennenzulernen.

Bücher aus dem Haus Schwabe sind sorgfältig gestaltet, fühlen sich gut an - und riechen wunderbar nach Druckerschwärze.

Schön, dass es das noch gibt!

\section{Lesung in Bern}

Daniel Lüthi stellt sein neues Buch in Bern an einer Lesung vor: Freitag, 25. November 2016, 19.30 Uhr

Buchhandlung LibRomania, Länggassstrasse 12, 3012 Bern Eintritt: $10 \mathrm{CHF}$ (inkl. Apéro)

Bitte reservieren: 0313053030

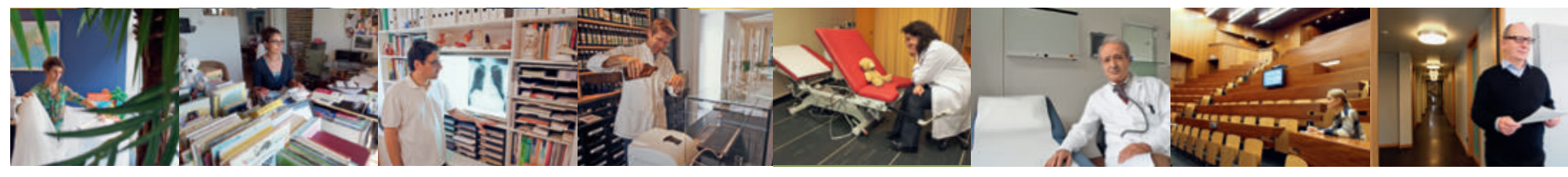

